\title{
Positive Effects of Soy Lecithin-Derived Phosphatidylserine plus Phosphatidic Acid on Memory, Cognition, Daily Functioning, and Mood in Elderly Patients with Alzheimer's Disease and Dementia
}

\author{
Margret I. Moré • Ulla Freitas • David Rutenberg
}

To view enhanced content go to www.advancesintherapy.com

Received: September 4, 2014 / Published online: November 21, 2014

(c) The Author(s) 2014. This article is published with open access at Springerlink.com

\section{ABSTRACT}

Introduction: We report previously unpublished, early pilot studies performed with a brain-health food supplement containing a proprietary blend of $100 \mathrm{mg}$ phosphatidylserine (PS) and $80 \mathrm{mg}$ phosphatidic acid (PA) produced from soy lecithin.

Methods: Serum analysis after single PS+PA ingestion was performed in healthy volunteers. A 3-month double-blind, placebocontrolled study assessed the influence of three PS+PA capsules/day, (300 mg PS + 240 mg PA/ day) or placebo on memory and mood in functioning, non-depressive elderly people with memory problems, using the Wechsler

Electronic supplementary material The online version of this article (doi:10.1007/s12325-014-0165-1) contains supplementary material, which is available to authorized users.

\section{I. Moré (ه)}

analyze \& realize $\mathrm{GmbH}$, Berlin, Germany

e-mail: mmore@analyze-realize.com

U. Freitas

Lonza Ltd., Basel, Switzerland

D. Rutenberg

Lipogen Ltd., Haifa, Israel
Memory Scale and the List of Depressive Symptoms. Furthermore, a 2-month randomized, double-blind, placebo-controlled trial assessed the effect of three PS $+\mathrm{PA}$ capsules/day (300 mg PS + $240 \mathrm{mg}$ PA/day) or placebo on daily functioning, mental health, emotional state, and self-reported general condition in patients with Alzheimer's disease (AD).

Results: Serum PS peaked $90 \mathrm{~min}$ after ingestion, returning to baseline after $180 \mathrm{~min}$. In the elderly, PS+PA [per protocol (PP) $n=31$ ], unlike placebo (PP $n=26$ ), significantly improved memory and prevented "winter blues" in a pre-post comparison. In the patients with $\mathrm{AD}$, daily functioning (i.e., 7 activities of daily living) under PS+PA (PP $n=53$ ) remained unchanged, but declined from 5.62 to 4.90 under placebo (PP $n=39$; $P=0.035)$, with significant group difference $(P=0.021)$. The PS+PA group had $3.8 \%$ deterioration and $90.6 \%$ stability in daily functioning, compared to $17.9 \%$ and $79.5 \%$ under placebo, respectively $(P=0.066)$. Fortynine percent of the PS+PA patients reported an improved general condition, compared to $26.3 \%$ under placebo $(P=0.084)$. 
Approximately, $43 \%$ of the PS+PA patients, but none under placebo, continued post-trial supplementation (while double-blinded). No negative side effects were observed.

Conclusion: PS is efficiently absorbed after oral consumption. A positive influence of PS+PA on memory, mood, and cognition was demonstrated among elderly test subjects. Short-term supplementation with PS+PA in patients with $\mathrm{AD}$ showed a stabilizing effect on daily functioning, emotional state and selfreported general condition. The data encourage long-term studies with $\mathrm{PS}+\mathrm{PA}$ in $\mathrm{AD}$ patients and other elderly with memory or cognition problems.

Keywords: Alzheimer's disease; Cognition; Daily functioning; Dementia; Elderly; Memory; MemreePlus $^{\mathrm{TM}}$; Mood; Phosphatidic acid; Soy lecithin-derived phosphatidylserine

\section{INTRODUCTION}

Cognitive impairment, such as memory loss (dementia), with increasing age is common and distressing to both the aged individual and their family [1-3]. Due to a gradual decline in cognitive capacity, patients with dementia are no longer able to continue their former regular occupational and social activities. Functional limitation restricts activities that are essential to daily living, such as bathing, dressing, toileting, and eating [4]. The physical and emotional impact of dementia caregiving is estimated to have resulted in healthcare costs of $\$ 9.1$ billion in the United States in 2012 [5].

A "normal", gradual, age-associated memory impairment (AAMI), with a decline of memory tasks by $50 \%$ or more over adulthood and aging, affects everybody [6]. Superimposed on this normal development, the majority of patients with dementia suffer from either a degenerative disease of the brain, mainly Alzheimer's disease (AD), or from multiple infarcts of the brain (vascular dementia), or from a combination of the two [6]. In a 65-year-old man, the remaining lifetime risk of $\mathrm{AD}$ has been shown to be $6.3 \%$, and the risk developing any dementing illness to be $10.9 \%$; with the corresponding risks for a 65 -year-old woman of $12 \%$ and $19 \%$, respectively [7].

The distress associated with memory impairments, including $\mathrm{AD}$, has ignited a search for viable treatments, and there have been various pharmacological or nutritional approaches explored to counter dementia $[8,9]$.

Age-related cognitive deficits have been linked to various mechanisms, including cumulative oxidative stress and age-related changes in cellular metabolism [10]. Many age-related neurochemical changes can be traced to structural and functional alterations in neuronal membranes [11]. A decline of phospholipids-particularly phosphatidylserine (PS) in neuronal membranes-has been associated with memory impairment and deficits in mental cognitive abilities $[12,13]$. This has led to the proposal that administration of endogenously occurring phospholipids may prevent or reverse age-related neurochemical deficits [14].

The phospholipids PS and phosphatidic acid (PA) are found in the neuronal membrane in high concentrations and are essential to its functioning, acting as a biological detergent, and keeping fatty substances soluble and cell membranes flexible $[15,16]$. PS was also found to reduce oxidative stress in the brain [17], as well as to stimulate neurotransmitter release $[18,19]$ and to increase brain glucose metabolism [20, 21]. Experiments with PSsupplemented rodents have shown an improvement in memory, learning capacity, and other cognitive parameters [19, 22-25]. 
Several human studies have shown the efficacy of bovine brain-derived PS in patients with dementia [26]. For example, AAMI subjects were supplemented by three-month administration of bovine brain-derived PS, $300 \mathrm{mg}$ daily [27]. An improvement on a number of neuropsychological tests was observed in the PS-supplemented subjects relative to those receiving placebo [27]. In a 12-week study with 149 AAMI patients, those supplemented with a bovine brain-derived PS formulation [100 mg PS three times daily (tid)] improved, relative to placebo, in daily life learning and memory tasks [28]. In another 6-month double-blind study, 494 cognitively impaired elderly were supplemented with $300 \mathrm{mg}$ bovine brain-derived PS daily (or placebo), resulting in a significant improvement in behavioral and cognitive parameters in the supplementation group [29].

In a different study, placebo-controlled supplementation of elderly with $100 \mathrm{mg}$ soy-PS six times daily ( $n=41$, PS-group) for 12 weeks did not reveal any significant cognitive improvements [30]. In contrast, a 12-week pilot study with 30 elderly volunteers suggested that soy lecithin-derived PS (100 mg tid) may have favorable effects on cognitive function in elderly with memory complaints [31]. Also, a different pilot study with 18 AAMI patients demonstrated significant positive effects on cognition [32]. A double-blind, randomized-controlled study investigating the effects of soy lecithin-derived PS on the cognitive functions of elderly Japanese subjects with memory complaints demonstrated (unlike placebo) significantly improved memory scores in PS-treated groups compared to baseline [33]. In different studies, PS-containing preparations were found to selectively dampen stress levels in humans [34-36], improve learning and perception parameters in children with attention-deficit hyperactivity disorder $[37$, 38], and improve the calculation speed of athletes [39].

Due to concerns regarding bovine spongiform encephalopathy, the use of PS (and PA) produced by enzymatic conversion of soybean lecithin $[40,41]$ is considered a safer alternative. Although PS from both sources can be considered safe [42, 43], currently, from a regulatory point of view, only the non-bovine brain-derived PS is allowed as a dietary supplement. The understanding of bovine brain-derived PS limitation initiated development of practical enzymatic conversion of soybean lecithin to PS and PA. The first company to commercially produce soy lecithin-derived PS was Lipogen Ltd. (Haifa, Israel) in 1991. The availability of soy lecithinderived PS further necessitated clinical testing of the new soy lecithin-derived PS in comparison to the old bovine brain-derived PS.

In the following, we describe pharmacokinetic data for soy lecithin-derived PS. Furthermore, the effect of dietary soy lecithin-derived PS + PA supplementation was evaluated in one uncontrolled and two placebo-controlled studies with elderly people with memory impairment or dementia, including $\mathrm{AD}$ (see Table 1 for an overview of the studies). The studies reported here were performed between 1992 and 1999 and were, to our knowledge, the first performed with plant-derived PS.

\section{METHODS}

\section{Kinetic Analysis of Soy Lecithin-Derived PS}

Standard soft gelatine capsules containing soy lecithin-derived PS and PA were supplied by Lipogen Ltd. (Haifa, Israel). The healthy 


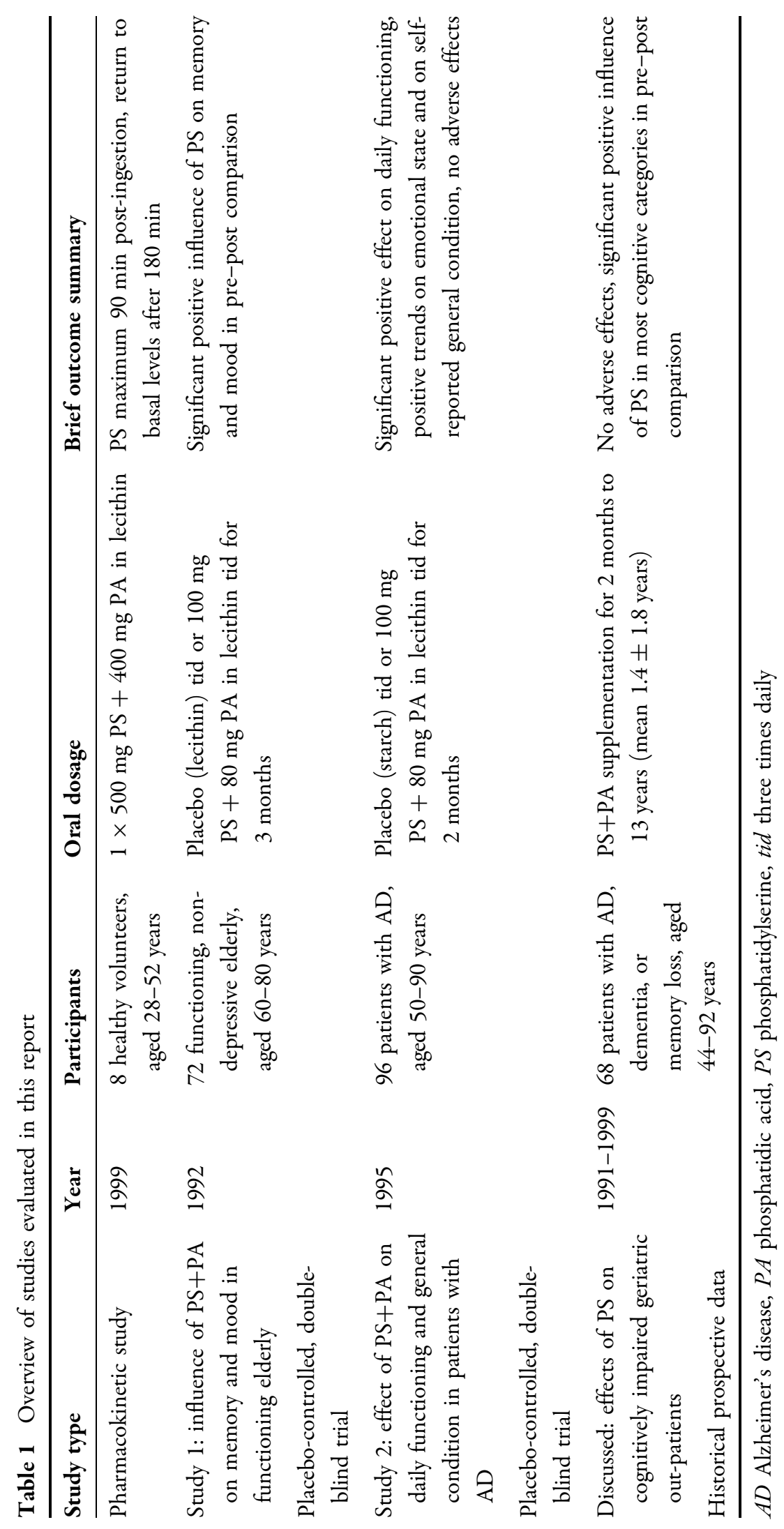


volunteers gave informed consent for participating in this study. The study complied with the Helsinki Declaration of 1996, however, an ethics committee vote was omitted since it was not mandatory at the time. After overnight fasting, healthy volunteers each received 5 standard soft gelatine capsules containing soy lecithin-derived PS and PA (100 mg PS + 80 mg $\mathrm{PA} /$ capsule) which were consumed at time 0 . Five milliliters of venous blood were drawn at time 0 and every 30 until $180 \mathrm{~min}$. The plasma was separated immediately and an exhaustive extraction of serum lipids was performed: $1 \mathrm{ml}$ of plasma was mixed with $9 \mathrm{ml}$ of isopropanol and then with $10 \mathrm{ml}$ of chloroform. The mixture was then centrifuged and the clear and homogeneous lipid extract was collected. It included approximately $\quad 100 \mu \mathrm{g} / \mathrm{ml}$ phospholipids.

Analysis of the individual phospholipids was carried out using two-dimensional thin-layer chromatography with chloroform, methanol, and ammonia (ratio 60:35:5, respectively) as first running solvent. Second running solvent was chloroform, acetic acid, acetone, and water (ratio 60:30:5:5, respectively). Scraped-off PL spots were processed for phosphorus analysis. The ratio of the phosphorus in the PS spot over the sum of phosphorus of all PL spots (defined as PS/PL) was taken as the measure of PS level in the plasma.

\section{Study 1: Effect of PS+PA on Memory and Mood in Functioning Elderly}

A 3-month study, performed in 1992 among elderly residents of three Israeli rural collective communities (Kibbutzim Givat Brenner, Gat, and Galon), assessed the effect of soy lecithinderived PS (100 mg) and PA (80 mg) tid on memory performance. The trial complied with the Declaration of Helsinki (1989 version); however, an ethics committee vote was not received and a trial registration was not performed as this was not mandatory at the time. Informed consent was obtained from all patients for being included in the study.

Participants were included according to the following criteria: Hebrew-speaking men and women aged 60-80 years who reported some memory problems in daily life; a score of at least 25 out of 30 on the Folstein Mini-Mental State Examination (MMSE) [44] (i.e., non-demented); a score of $<17$ on the List of Depressive Symptoms (LDS) [45], indicating that the subject was not depressed; not suffering from any active or recent major disease; and not taking medication that could influence memory. Recruitment was accomplished in coordination with the kibbutz health committees and clinics. The nature of the study was explained to the elderly at special meetings.

The primary outcome measure was a score change in the Wechsler Memory Scale (WMS; without age correction) score [46]. The test examines memory, learning, association, and information gathering as distinct from intelligence [47]. The components of the test are orientation, information, concentration (counting backwards 20-1), saying alphabet backwards, adding by 3 's up to 40 , repeating short stories, repeating numbers forward and backward, learning word associations, and visual memory.

As an additional outcome variable, mood was tested using the LDS [45], which contains 22 items expressing negative affect and 12 items with positive affect. The resulting score was also used to exclude those with a score of $<17$ during recruitment.

Baseline measures included demographic, social, functioning, and health data, as well as the total number of years of education. Health variables included diseases and medication use. 
Participants were randomly assigned to either receive placebo (500 $\mathrm{mg}$ lecithin) or $100 \mathrm{mg}$ of PS $+80 \mathrm{mg}$ PA mixed with lecithin tid for 3 months (L-Telect, Lipogen Ltd., Haifa, Israel; similar to current MemreePlus ${ }^{\mathrm{TM}}$, Lonza Ltd., Basel, Switzerland).

\section{Study 2: Effect of PS+PA on Daily Functioning and General Condition in 96 Patients with AD}

A 2-month study, performed in 1995, was designed as a double-blind, randomized, placebo-controlled trial. Patients with AD were recruited from the Kaplan Medical Centre (Rehovot, Israel), from kibbutzim near Rehovot, and the Israel Association of Alzheimer's Disease. Study substances and procedures, as well as the informed consent form, complied with the Declaration of Helsinki (1989 version); however, an ethics committee vote was not received and a trial registration was not performed, as this was not mandatory at the time. All participants or surrogates had signed a written informed consent prior to the beginning of the trial.

Participants were included according to the following criteria: aged 50-90 years; diagnosed with definite-probable AD (Alzheimer's Disease and Related Disorders Association); history of dementia >6 months; reported level of functioning limitations or decline; no acute or chronic dysfunction in a major organ system; no endocrine disorder; no chronic renal or hepatic failure; no multi-infarct dementia; no dementia due to Parkinson's, Pick, or Creutzfeldt-Jakob disease; and no suspected reversible dementia.

Without wash-out of possible previous medications, 56 patients $(58.3 \%)$ were orally supplemented with $100 \mathrm{mg}$ of PS $+80 \mathrm{mg}$ PA mixed with lecithin tid (L-Telect); 40 patients (41.7\%) received placebo (starch).
Baseline and 2-month examinations included general self-reporting and scoring of activities of daily living (7-ADL) items [daily functioning index from 0 to 7 with a point for each independent task (bathing, dressing, grooming, toileting, feeding, transferring, and walking; 0 points means a failure in all tasks)] [48]. The mental status was assessed by Folstein MMSE [44], while mood was assessed by a simplified Tel-Aviv University Rosen Target Detection (RTD) test [45]. One-month posttrial, still double-blinded, accessible patients/ families were asked by telephone whether they continued to use the product at their own expense.

\section{Statistical Analysis}

Data analysis for both studies was done using the SPSS statistical package (IBM Corporation, Armonk, NY, USA). For Study 1, all $P$ values are two-tailed unless otherwise indicated. The influence of the main variables was also estimated by the Pearson chi-square test for categorical data. Both stepwise linear regression and multiple linear regression were performed to estimate the joint effect of the variables.

For Study 2, distribution of all variables was analyzed for outliers. Selected pairs of variables were tested for the presence of incompatible combinations of values. The comparability of groups was tested using $t$ test for continuous variables and crosstabulation chi-square for discrete variables. Comparison of effects in two groups was performed for each parameter separately using the following methods: independent samples $t$ test for after-before difference; analysis of variance in two groups with "after" values as an outcome and "before" values as a covariate. 


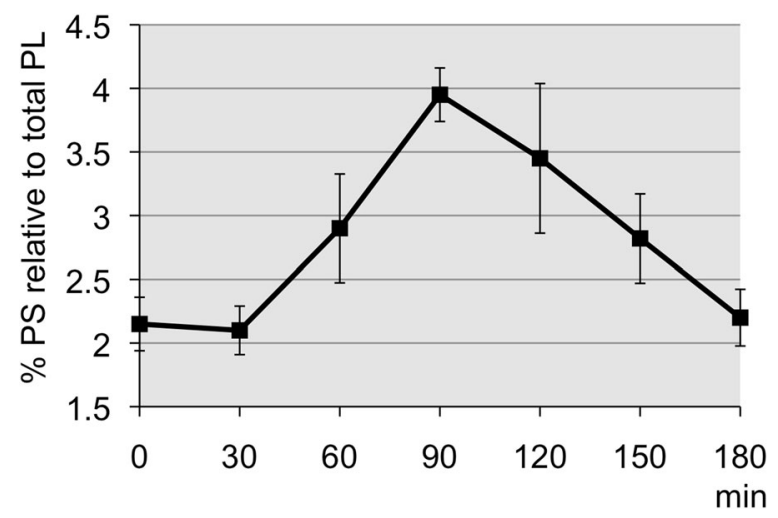

Fig. 1 Overall average profile of serum PS relative to total serum PL in eight healthy volunteers after single ingestion of five capsules of soy lecithin-derived PS + phosphatidic acid. PL Phospholipids, PS phosphatidylserine

\section{RESULTS}

\section{Kinetic Analysis of Soy Lecithin-Derived PS}

Serum analysis of eight overnight-fasted healthy volunteers (aged 28-52 years) after ingestion of soy lecithin-derived PS+PA was performed to assess the PS kinetics. The serum level of PS in relation to the level of total serum phospholipids before the intake was in the range of $1.8-2.2 \%$, but it started increasing $30 \mathrm{~min}$ after ingestion, with a peak value 90 min after intake and returned to basal level 180 min after intake (Fig. 1). The soy lecithinderived PS+PA was well-tolerated, with no side effects reported.

\section{Study 1: Effect of PS+PA on Memory and Mood in Functioning Elderly}

\section{Study Population}

Out of a total of 414 kibbutz members aged 60-80 years, 102 (24.6\%) elderly reported some memory difficulties. After invoking the inclusion criteria, a total of 72 subjects of both sexes were included in the study, after they had submitted a written consent (40 PS+PA, 32 placebo). These non-depressed elderly with memory problems performed the following tests at baseline and after 3 months of PS+PA $(n=40)$ or placebo $(n=32)$ supplementation: (a) the WMS examined memory, learning, association, and information gathering; (b) mood was tested using the LDS.

At the end of 3 months, 57 participants could be retested (Fig. 2, randomization scheme; Table 2, characteristics of study population). Fifteen subjects could not be located or dropped out due to death, non-compliance, or surgery. There were no statistically significant differences between the dropouts of the placebo group and the study group in any dimension examined. The characteristics of the placebo and supplementation groups are shown in Table 2. The groups were found to be statistically comparable.

\section{The WMS}

Figure 3 a shows that there was a significant testretest improvement in total WMS scores for both the PS+PA group and the placebo group. The changes were significant in both the placebo group $(P<0.013)$ and the $\mathrm{PS}+\mathrm{PA}$ group $(P<0.005)$.

Although no significant differences were found when comparing the scores between groups, further subgroup analysis shows that the initial WMS score had a significant effect on the change in the WMS score (Fig. 3b). For those with a low initial score $(<69)$, there was no significant difference between groups $(P<0.91)$. However, for those with a higher initial WMS score $(>68)$, those who received a placebo actually experienced a small score decrease after 3 months, whereas those who received $\mathrm{PS}+\mathrm{PA}$ experienced a significant score increase; accordingly, there was a significant difference between groups $(P<0.03)$. 

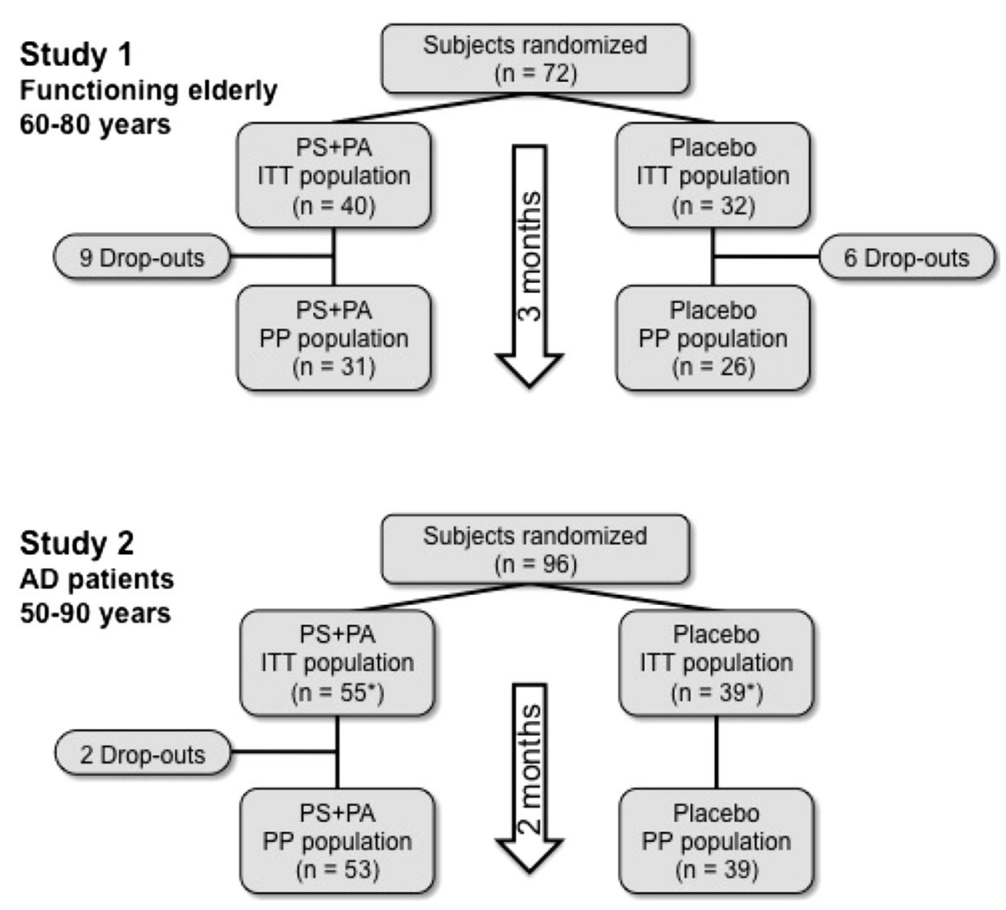

Fig. 2 Schematic overview of the two double-blinded, placebo-controlled studies. Asterisks one subject of each group dropped out between allocation to the study group

Regression analysis indicates that there was a significantly greater effect of PS+PA supplementation on those with high initial WMS scores than on those with low initial WMS scores.

An analysis of the nine components that constitute the WMS score was performed: in the placebo group, a significant increase in the WMS score was found in the total score only (3.3\%, $P<0.03)$. However, in the PS+PA group, there was a highly significant increase not only in the total score $(3.5 \%, P<0.001)$ but also in the test's components: information $(2.2 \%$, $P<0.04)$, visual memory $(10.6 \%, P<0.01)$, and memorizing numbers $(7.0 \%, P<0.01)$.

\section{Mood Changes: LDS}

To assess mood changes after PS+PA administration, the score on the LDS was assessed before and after supplementation and start of supplementation. $A D$ Alzheimer's disease, ITT intention to treat, $P A$ phosphatidic acid, $P S$ phosphatidylserine, $P P$ per protocol

(Fig. 3c). In a pre-post comparison, the results show that the placebo group experienced a significant increase in depressive symptoms between baseline (late summer) and the second time point (winter; $P<0.001$ ), whereas the PS + PA group revealed no significant change $(P<0.4)$.

\section{Study 2: Effect of PS+PA on Daily Functioning and General Condition in Patients with AD}

\section{Study Population}

Sixty-one women (63.5\%) and 35 men with a mean age of 75.3 years and with $\mathrm{AD}$ participated in a study testing the effects of soy lecithin-derived PS+PA on memory and mood; one subject of each group dropped before supplementation started, and another 2 subjects from the PS+PA group dropped out 
Table 2 Study population of the placebo-controlled studies

\begin{tabular}{lll}
\hline $\begin{array}{l}\text { Study 1: functioning elderly, } \\
\text { aged 60-80 years }\end{array}$ & \multicolumn{2}{l}{ PP population } \\
\cline { 2 - 3 } $\begin{array}{l}\text { Mean age, years }( \pm S D) \\
\begin{array}{l}\text { Puration of education, years } \\
( \pm S D)\end{array}\end{array}$ & $71.4 \pm 6.2$ & $70.8 \pm 7.0$ \\
Male & $8(26 \%)$ & $11(42 \%)$ \\
Female & $23(74 \%)$ & $15(58 \%)$ \\
Concomitant medication & $12(39 \%)$ & $13(50 \%)$ \\
$1-2$ diseases & $14(45 \%)$ & $10(39 \%)$ \\
$\geq 3$ diseases & $5(16 \%)$ & $8(31 \%)$ \\
Total & 31 & 26 \\
\hline
\end{tabular}

\begin{tabular}{lll}
\hline $\begin{array}{l}\text { Study 2: patients with AD, } \\
\text { aged 50-90 years }\end{array}$ & \multicolumn{2}{l}{ ITT population } \\
\cline { 2 - 3 } & PS+PA & Placebo \\
\hline Aged 50-65 years & $8(14.5 \%)$ & $6(15.4 \%)$ \\
Aged 66-80 years & $29(52.7 \%)$ & $17(43.5 \%)$ \\
Aged $81-90$ years & $18(32.7 \%)$ & $16(41.1 \%)$ \\
High education ( $\geq 13$ years) & $14(25.5 \%)$ & $5(12.8 \%)$ \\
Male & $23(41.8 \%)$ & $11(28.2 \%)$ \\
Female & $32(58.2 \%)$ & $28(71.8 \%)$ \\
$0-1$ diseases & $23(41.8 \%)$ & $11(28.2 \%)$ \\
$\geq 2$ diseases & $32(58.2 \%)$ & $28(71.8 \%)$ \\
Total & 55 & 39
\end{tabular}

Values are presented as $n$ (\%) unless otherwise stated $A D$ Alzheimer's disease, ITT intention to treat, $P A$ phosphatidic acid, $P S$ phosphatidylserine, $P P$ per protocol

during supplementation (Fig. 2, randomization scheme; Table 2, baseline characteristics of ITT population).

At baseline, $29.8 \%$ of the participants had mild dementia (based on MMSE score), 21.2\% were at the moderate stage, and $12.8 \%$ at the severe stage. $36.2 \%$ were categorized as borderline (31 of them scored 24-26 points), with a clinical diagnosis of AD made. At study onset, $30 \%$ of the participants reported dizziness, 23\% reported sleep disorders, and $20 \%$ reported constipation. The characteristics of the two randomized groups suggested that they were comparable.

The analyzed PP population was 39 people taking placebo, and 53 people taking PS + PA.

\section{7-ADL Functioning Index}

A comparison of the assessments of the 7-ADLs functioning index (Table 3) revealed no progression of the deterioration (stability) in the PS+PA group, compared to a decline in the control group: from 5.62 to 4.90 $(P=0.039)$. The statistical significance of the difference between the groups by this measurement increased from $P=0.433$ before the supplementation to $P=0.021$ afterwards.

A positive effect could also be found in 7-ADL functioning change patterns rates (Table 3): $3.8 \%$ of deterioration (2 of 53) in the PS+PA group compared to $17.9 \%$ (7 of 39 ) in placebo, and $90.6 \%$ of stability (48 of 53 ) versus $79.5 \%$ (31 of 39$)$ in the control group $(P=0.066)$.

\section{Mental State (Folstein MMSE)}

Examination of mental state (Folstein MMSE) change patterns revealed a slight and nonsignificant improvement in both groups $(P=0.872$; Fig. $4 a)$. Deterioration and stability rates were slightly better in the PS+PA group. However, it should be noted that 12 of 51 $(23.5 \%)$ patients in the PS+PA group progressed from abnormal $(\leq 23)$ initial scores to normal $(<23)$ after supplementation, compared to 4 of 35 (11.4\%) in the control group.

\section{RTD Scale}

A change pattern comparison of the means of the index of emotional state (RTD scale) indicated minor but insignificant changes 

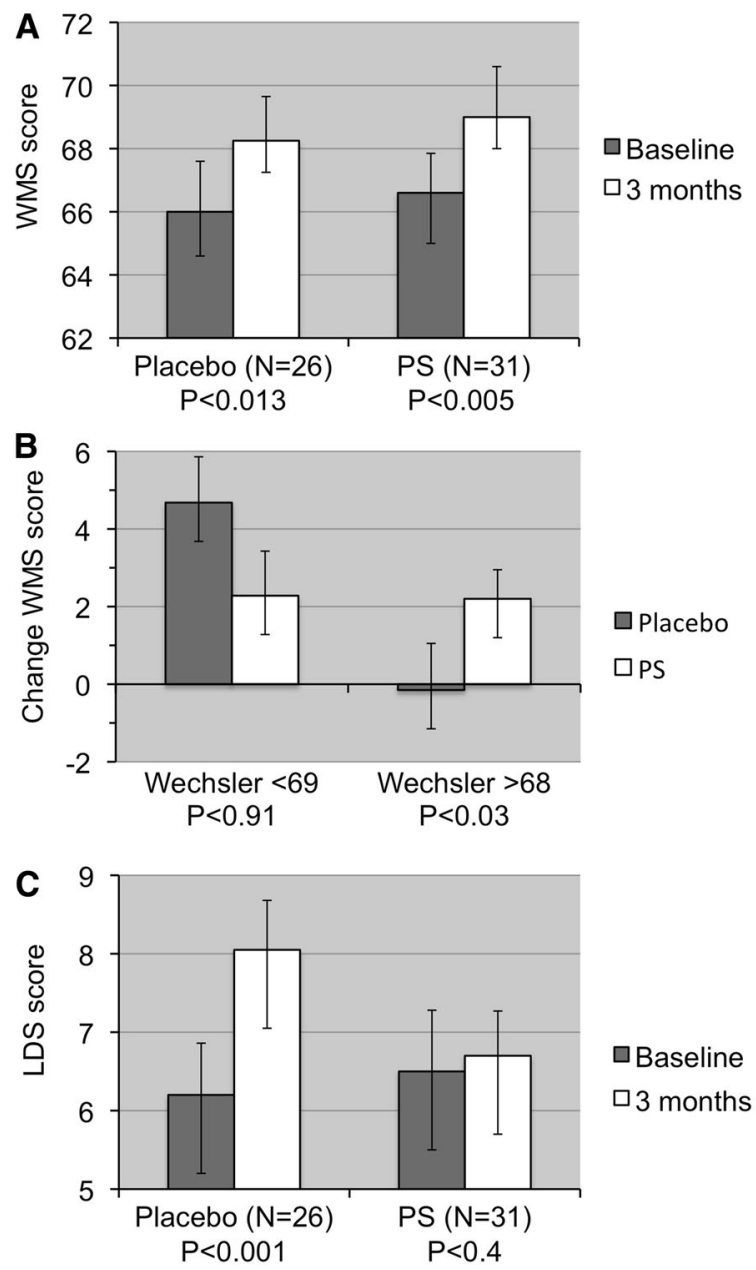

Fig. 3 WMS score results. Mean \pm SEM and $P$ values for one-tailed $t$ test are indicated. a Test score before and after 3 months of PS+PA supplementation. $\mathbf{b}$ Change in WMS score after 3 months of PS+PA supplementation by baseline level (T1). c Depressive symptoms score before and after 3 months of PS+PA supplementation. LDS List of Depressive Symptoms, PA phosphatidic acid, PS phosphatidylserine, WMS Wechsler Memory Scale

$(P=0.156$; Fig. $4 b)$. However, trends could be observed: comparing the PS+PA group with the placebo group, there were higher rates of stability (32.7\% vs. $24.2 \%$, respectively) and improvement (44.9\% vs. $33.3 \%$, respectively). Also, the deterioration was only approximately half as severe with $22.4 \%$ vs. $42.4 \%$, respectively.

\section{Self-Reported General Condition}

The assessment of the self-reported general condition showed that $49.0 \%$ of patients in the PS+PA group reported an improvement in their general condition compared to $26.3 \%$ in placebo group ( $P=0.084$; Fig. $4 \mathrm{c})$.

\section{Post-Trial Consumption Rate}

The behavioral indicator of post-trial consumption rates revealed a large difference between the groups: $42.9 \%$ of the patients in the PS+PA group (6 of 14 assessed patients) decided to continue the supplementation at their own expense, in contrast to 0 of 11 in the placebo group $(P=0.010)$.

\section{Safety and Tolerability}

Regarding tolerability, $88.2 \%$ of the patients in PS+PA group and $91.1 \%$ of placebo group reported having no side effects at all. The remaining patients could not remember or had various sensations, most of which they could not relate directly to the supplementation.

\section{Historical Prospective Study: Effect} of PS+PA on Cognitive Abilities of Patients with AD, other Dementia, or Memory Loss

Historical prospective data of out-patients of two medical centers in Israel were analyzed for the effects of PS+PA on the cognitive abilities of 68 cognitively impaired elderly suffering from $\mathrm{AD}$ (76.1\%), other dementia (14.9\%), or memory loss $(8.9 \%)$. Most of the patients had a moderate degree of dementia. Mean supplementation with the soy lecithin-derived PS + PA was for $1.4 \pm 1.8$ years (range 2 months13 years). Either no change or an improvement after 3-6 months as well as after 12 months of PS+PA supplementation could be demonstrated 
Table 3 Comparison of 7-ADL functioning index (Study 2)

\begin{tabular}{llll}
\hline & PS+PA & Placebo & P value \\
\hline Before-after comparison & & \\
Baseline & $6.23 \pm 1.98$ & $5.62 \pm 2.42$ & 0.433 \\
After 2 months & $6.23 \pm 2.19$ & $4.90 \pm 3.00$ & 0.021 \\
Difference & $0.00 \pm 0.52$ & $-0.72 \pm 2.05$ & 0.039 \\
Change pattern analysis & & \\
Deterioration & $3.8 \%$ & $17.9 \%$ & \\
Stability & $90.6 \%$ & $79.5 \%$ & 0.066 \\
Improvement & $5.6 \%$ & $2.6 \%$ & \\
\hline
\end{tabular}

7- $A D L 7$ activities of daily living, $P A$ phosphatidic acid, $P S$ phosphatidylserine

(see Fig. S1 in the electronic supplementary material). A statistically significant positive influence of PS+PA supplementation could be recorded in most categories with patients suffering from cognitive impairments.

\section{DISCUSSION}

PS is a structural PL found in neuronal membrane and is essential to its functioning $[15,16]$. In various studies, PS (mostly derived from bovine brain) was found to improve cognitive ability and functioning in demented patients (see "Introduction"). However, the use of PS from a vegetative source, free of risk of encephalopathy, is considered advantageous, and the presented human studies were, to the best of our knowledge, the first to use plantderived PS.

The kinetic analysis in healthy volunteers demonstrated that soy lecithin-derived PS is absorbed and metabolized, with elevated serum levels for at least $1.5 \mathrm{~h}$ after an oral dose.

In Study 1 on 72 non-demented, nondepressed, functioning elderly with various degrees of AAMI, the post-supplementation memory testing scores in the PS+PA supplementation group were significantly higher than baseline, with a higher degree of significance compared to the placebo group. Furthermore, when pre- and postsupplementation results of components of the memory test were compared, none of the placebo group's changes were statistically significant, whereas results for maintaining information, visual memory, and memorizing numbers improved with statistical significance of $P<0.05$ within the PS+PA group. The nonsignificant improvement trends observed in the placebo group may be explained as a learning (test-retest) effect, and/or with the $4-8 \%$ of PA also contained within soy lecithin [49].

Comparing both groups, we found that subjects who received PS+PA supplementation and who had scored high on the initial WMS showed more significant memory improvements, whereas those who had scored low did not. A probable explanation may be the rather small size of the study, as well as its relatively short duration. PS+PA was also observed to have clear beneficial effects in patients with dementia, especially if the expected gradual cognitive deterioration is taken into consideration:

In the placebo-controlled, randomized study involving patients with AD (Study 2), a statistically significant positive effect of PS $+\mathrm{PA}$ was found on the 7-ADLs functioning index, while a significantly more pronounced deterioration was revealed in the placebo group.

Functioning is considered to be highly important in geriatric practice, since it is strongly associated with pathology, and is a determinant of measurable outcomes, such as morbidity, mortality, hospitalization, and costs, as well as efficacy of interventions and treatments. The association of functioning 

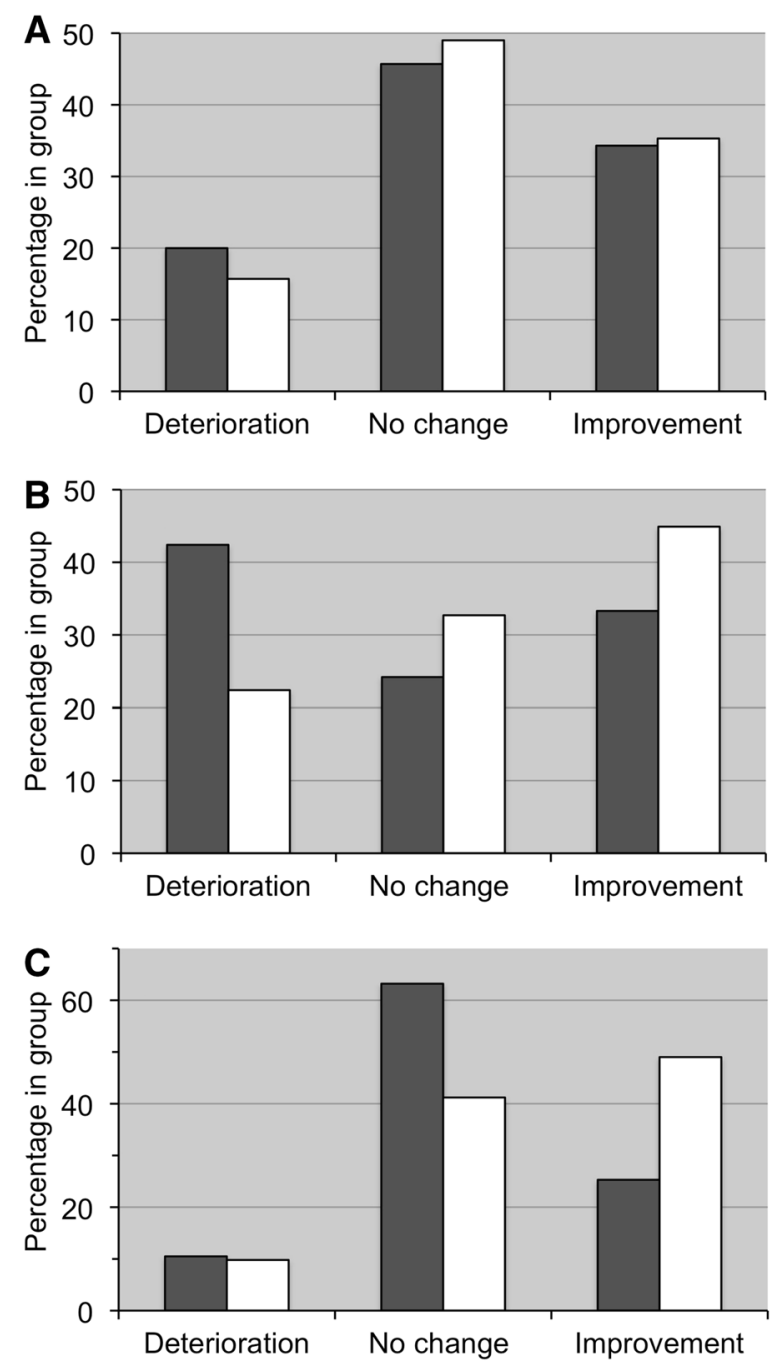

Fig. 4 Change patterns of mental state, emotional state, and self-reported general condition. The percentage of patients who experienced deterioration, no change, or improvement after 2 months of PS+PA supplementation (white bars) or placebo (black bars) is indicated. a Mental state (Folstein Mini-Mental State Examination); $P=0.872$. b Index of emotional state (Rosen Target Detection scale); $P=0.156$. c Self-reported general condition; $P=0.084$. $P A$ phosphatidic acid, $P S$ phosphatidylserine

with quality of life and need of care is well established [50].

The MMSE has a rather low sensitivity to minor changes [51]; accordingly, only slight positive trends after the supplementation of PS+PA could be observed after 2 months. However, the positive trends of PS+PA supplementation on change patterns of functioning and self-reported general condition are encouraging. Significance could possibly not be reached due to the short study duration and the limited sample size.

The data from Study 2 is complemented by the historical prospective data from 68 cognitively impaired elderly dementia patients-especially memory categories and organization skills appeared to improve with PS+PA in pre-post comparison.

The superiority of the PS+PA supplementation can also be derived from the fact that 6 out of $14(42.9 \%)$ patients (or their families) in the PS+PA supplementation group of Study 2 (while still double-blinded) decided to continue the supplementation at their own expense after study termination, compared to none in the placebo group. Similarly, many of those patients with dementia analyzed in a historical prospective manner continued PS+PA supplementation at their own expense for more than a year (data not shown).

In addition to the positive influence on the memory, the study on functioning elderly reveals a positive effect of PS+PA on mood. None of the participants in Study 1 had been initially depressed, since high LDS scores were excluded. Nevertheless, a worsening of mood with time was observed within the placebo group, which was not seen in the PS+PA supplementation group. "Winter blues", also known as seasonal affective disorder or seasonal depression, is a universal phenomenon in higher latitudes [52, 53]. Since this study started in late summer and ended in winter, we suggest that soy lecithinderived PS+PA may be advantageous in prevention of "winter blues" and depression. Maggioni et al. [54] reported a positive effect of PS on geriatric depression. 
These results go along with strong trends from Study 2 on patients with AD. There were nearly twice as many patients with emotional decline in the placebo group compared to the PS+PA supplementation group, with higher rates of improvement in the $\mathrm{PS}+\mathrm{PA}$ group. Dementia and mood are highly related, and sometimes dementia is accompanied by depression, thus the effects of PS on mood may be indirect effects due to the positive impact on cognition and memory.

\section{CONCLUSION}

Altogether our results suggest that PS $+\mathrm{PA}$ can support memory and cognitive function, which is especially apparent in those who have developed cognition deficiencies. Further investigations should assess a larger sample for a longer duration, so that the full and prolonged effects of PS+PA on cognition, memory, daily functioning, and mood in elderly and patients with dementia can be more thoroughly determined.

\section{ACKNOWLEDGMENTS}

We are grateful to all participants of this study. These studies were funded by Lipogen Ltd. (Haifa, Israel). Article processing charges were funded by Lonza Ltd. (Basel, Switzerland). The first author evaluated the original research data and wrote this manuscript. The third author provided the research data and the second author helped finalize the manuscript. All named authors meet the ICMJE criteria for authorship for this manuscript, take responsibility for the integrity of the work as a whole, and have given final approval for the version to be published. All authors had full access to all of the data in this study and take complete responsibility for the integrity of the data and accuracy of the data analysis.

Conflict of interest. The studies reported here were conducted with products by Lipogen Ltd. (Haifa, Israel), which are also distributed by Lonza Ltd. (Basel, Switzerland), which has organized this publication. M. I. Moré, who has a long scientific background, is an employee of analyze and realize $\mathrm{GmbH}$, Berlin, Germany, a company which also offers medical writing services. U. Freitas is an employee of Lonza Ltd. D. Rutenberg is an employee of Lipogen Ltd.

Compliance with ethics guidelines. All procedures followed were in accordance with the ethical standards of the Helsinki Declaration of 1975, as revised in 1989 (and as revised in 1996 for the pharmacokinetic studies performed in 1999). Informed consent was obtained from all patients for being included in the studies. However, an ethics committee vote was not received and a trial registration was not performed, as this was not mandatory at the time.

Open Access. This article is distributed under the terms of the Creative Commons Attribution Noncommercial License which permits any noncommercial use, distribution, and reproduction in any medium, provided the original author(s) and the source are credited.

\section{REFERENCES}

1. Cutler SJ, Grams AE. Correlates of self-reported everyday memory problems. J Gerontol. 1988;43:S82-90.

2. Khan ZU, Martín-Montañez E, Navarro-Lobato I, Muly EC. Memory deficits in aging and neurological diseases. Prog Mol Biol Transl Sci. 2014;122:1-29. 
3. Nolan KA, Blass JP. Preventing cognitive decline. Clin Geriatr Med. 1992;8:19-34.

4. Wan T, Odell B. Factors affecting the use of social and health services for the elderly. Aging Society. 43, 153-161.

5. Alzheimer's Association, 2013. 2013 Alzheimer's disease facts and figures. http://www.alz.org/ downloads/facts_figures_2013.pdf. Accessed 31 Oct 2014.

6. Crook TH, West RL. Name recall performance across the adult life-span. Br J Psychol. 1990;81:335-49.

7. Seshadri S, Wolf PA, Beiser A, et al. Lifetime risk of dementia and Alzheimer's disease. The impact of mortality on risk estimates in the Framingham Study. Neurology. 1997;49:1498-504.

8. Kidd PM. Alzheimer's disease, amnestic mild cognitive impairment, and age-associated memory impairment: current understanding and progress toward integrative prevention. Altern Med Rev. 2008;13:85-115.

9. Wollen KA. Alzheimer's disease: the pros and cons of pharmaceutical, nutritional, botanical, and stimulatory therapies, with a discussion of treatment strategies from the perspective of patients and practitioners. Altern Med Rev. 2010;15:223-44.

10. Billard JM. Serine racemase as a prime target for agerelated memory deficits. Eur J Neurosci. 2013;37:1931-8.

11. Sun A, Sun G. Neuroechemical aspects of the membrane hypothesis of aging. Interdiscip Top Gerontol. 1979;15:34-53.

12. Oma S, Mawatari S, Saito $\mathrm{K}$, et al. Changes in phospholipid composition of erythrocyte membrane in Alzheimer's disease. Dement Geriatr Cogn Disord Extra. 2012;2:298-303.

13. Schroeder F. Role of membrane lipid asymmetry in aging. Neurobiol Aging. 1984;5:323-33.

14. Calderini $G$, et al. Phospholipids as a pharmacological tool in the aging brain. In: Horrocks L, Kanfer J, Porcellati G, editors. Phospholipids in the nervous system, physiological roles. Vol. 2. New York: Raven Press; 1985. p. 11-19.

15. Mozzi R, Buratta S, Goracci G. Metabolism and functions of phosphatidylserine in mammalian brain. Neurochem Res. 2003;28:195-214.

16. Thorne Research I. Phosphatidylserine. Altern Med Rev. 2008;13:245.
17. Chaung HC, Chang CD, Chen PH, Chang CJ, Liu $\mathrm{SH}$, Chen CC. Docosahexaenoic acid and phosphatidylserine improves the antioxidant activities in vitro and in vivo and cognitive functions of the developing brain. Food Chem. 2013;138:342-7.

18. Nishizuka Y. Turnover of inositol phospholipids and signal transduction. Science. $1984 ; 225: 1365-70$.

19. Suzuki S, Yamatoya H, Sakai M, Kataoka A, Furushiro M, Kudo S. Oral administration of soybean lecithin transphosphatidylated phosphatidylserine improves memory impairment in aged rats. J Nutr. 2001;131:2951-6.

20. Heiss WD, Kessler J, Mielke R, Szelies B, Herholz K. Long-term effects of phosphatidylserine, pyritinol, and cognitive training in Alzheimer's disease. A neuropsychological, EEG, and PET investigation. Dementia. 1994;5:88-98.

21. Klinkhammer P, Szelies B, Heiss W-D. Effect of phosphatidylserine on cerebral glucose metabolism in Alzheimer's disease. Dementia. 1990;1:197-201.

22. Blokland A, Honig W, Brouns F, Jolles J. Cognitionenhancing properties of subchronic phosphatidylserine (PS) treatment in middle-aged rats: comparison of bovine cortex PS with egg PS and soybean PS. Nutrition. 1999;15:778-83.

23. Furushiro M, Suzuki S, Shishido Y, et al. Effects of oral administration of soybean lecithin transphosphatidylated phosphatidylserine on impaired learning of passive avoidance in mice. Jpn J Pharmacol. 1997;75:447-50.

24. Lee B, Sur BJ, Han JJ, et al. Krill phosphatidylserine improves learning and memory in Morris water maze in aged rats. Prog Neuropsychopharmacol Biol Psychiatry. 2010;34:1085-93.

25. Zanotti A, Valzelli L, Toffano G. Chronic phosphatidylserine treatment improves spatial memory and passive avoidance in aged rats. Psychopharmacology. 1989;99:316-21.

26. Amaducci L, Crook TH, Lippi A, et al. Use of phosphatidylserine in Alzheimer's disease. Ann N Y Acad Sci. 1991;640:245-9.

27. Villardita C, Grioli S, Salmeri G, et al. Multicentre clinical trial of brain phosphatidylserine in elderly patients with intellectual deterioration. Clin Trials J. 1987;24:84-93.

28. Crook TH, Tinklenberg J, Yesavage J, Petrie W, Nunzi MG, Massari DC. Effects of phosphatidylserine in age-associated memory impairment. Neurology. 1991;41:644-9. 
29. Cenacchi T, Bertoldin T, Farina C, Fiori MG, Crepaldi G. Cognitive decline in the elderly: a double-blind, placebo-controlled multicenter study on efficacy of phosphatidylserine administration. Aging. 1993;5:123-33.

30. Jorissen BL, Brouns F, Van Boxtel MP, et al. The influence of soy-derived phosphatidylserine on cognition in age-associated memory impairment. Nutr Neurosci. 2001;4:121-34.

31. Richter Y, Herzog Y, Lifshitz Y, Hayun R, Zchut S. The effect of soybean-derived phosphatidylserine on cognitive performance in elderly with subjective memory complaints: a pilot study. Clin Interv Aging. 2013;8:557-63.

32. Schreiber S, Kampf-Sherf O, Gorfine M, Kelly D, Oppenheim Y, Lerer B. An open trial of plant-source derived phosphatydilserine for treatment of agerelated cognitive decline. Isr J Psychiatry Relat Sci. 2000;37:302-7.

33. Kato-Kataoka A, Sakai M, Ebina R, Nonaka C, Asano $\mathrm{T}$, Miyamori T. Soybean-derived phosphatidylserine improves memory function of the elderly Japanese subjects with memory complaints. J Clin Biochem Nutr. 2010;47:246-55.

34. Hellhammer J, Fries E, Buss C, et al. Effects of soy lecithin phosphatidic acid and phosphatidylserine complex (PAS) on the endocrine and psychological responses to mental stress. Stress. 2004;7:119-26.

35. Hellhammer J, Hero $\mathrm{T}$, Franz $\mathrm{N}$, Contreras $\mathrm{C}$, Schubert M. Omega-3 fatty acids administered in phosphatidylserine improved certain aspects of high chronic stress in men. Nutr Res. 2012;32:241-50.

36. Hellhammer J, Vogt D, Franz N, Freitas U, Rutenberg D. A soy-based phosphatidylserine/ phosphatidic acid complex (PAS) normalizes the stress reactivity of hypothalamus-pituitary-adrenalaxis in chronically stressed male subjects: a randomized, placebo-controlled study. Lipids Health Dis. 2014;13:121.

37. Hirayama S, Terasawa K, Rabeler R, et al. The effect of phosphatidylserine administration on memory and symptoms of attention-deficit hyperactivity disorder: a randomised, double-blind, placebocontrolled clinical trial. J Hum Nutr Diet. 2014;27(Suppl 2):284-91.

38. Manor I, Magen A, Keidar D, et al. The effect of phosphatidylserine containing Omega3 fattyacids on attention-deficit hyperactivity disorder symptoms in children: a double-blind placebocontrolled trial, followed by an open-label extension. Eur Psychiatry. 2012;27:335-42.
39. Parker AG, Gordon J, Thornton A, et al. The effects of IQPLUS Focus on cognitive function, mood and endocrine response before and following acute exercise. J Int Soc Sports Nutr. 2011;8:16.

40. Damnjanovic J, Iwasaki Y. Phospholipase D as a catalyst: application in phospholipid synthesis, molecular structure and protein engineering. J Biosci Bioeng. 2013;116:271-80.

41. Eibl H, Kovatchev S. (53) Preparation of phospholipids and their analogs by phospholipase D. In: Lowenstein $\mathrm{J}$, editor. Methods in enzymology, volume 72: lipids, part D. New York: Academic Press; 1981.

42. Jorissen BL, Brouns F, Van Boxtel MP, Riedel WJ. Safety of soy-derived phosphatidylserine in elderly people. Nutr Neurosci. 2002;5:337-43.

43. Vakhapova V, Richter Y, Cohen T, Herzog Y, Korczyn AD. Safety of phosphatidylserine containing omega-3 fatty acids in non-demented elderly: a double-blind placebo-controlled trial followed by an open-label extension. BMC Neurol. 2011;11:79.

44. Folstein M, Folstein S, McHugh P. Mini mental state; a practical method for grading the cognitive state of patients for the clinician. J Psychiatr Res. 1975;12:189-98.

45. Tel-Aviv University-Psychology. List of depressive symptoms (LDS). Dept of Psychology, Unit of Adulthood and Aging, Tel-Aviv University; 1981.

46. Wechsler DA. Standardized memory scales for clinical use. J Psychol. 1945;19:87-95.

47. Kane R, Kane R. Assessing the elderly; a practical guide to measurement. Lexington: Lexington Books; 1981.

48. Walter-Ginzburg A. Factors predicting health care utilisation among the community dwelling oldestold in Israel. Lecture at the 45 Annual Scientific Meeting of the Gerontological Society of America, Washington, DC. 1992.

49. Szuhaj B. Lecithins. In: Bailey's industrial oil and fat products. Vol. 3:13; 2005.

50. Forbes D, et al. Exercise programs for people with dementia. Sao Paulo Med J. 2014;132:195-6.

51. Tombaugh TN, McIntyre NJ. The mini-mental state examination: a comprehensive review. J Am Geriatr Soc. $1992 ; 40: 922-35$.

52. Booker JM, et al. Seasonal depression and sleep disturbances in Alaska and Siberia: a pilot study. Arct Med Res. 1991;Suppl:281-4. 
53. Yang AC, et al. Do seasons have an influence on the incidence of depression? The use of an internet search engine query data as a proxy of human affect. PLoS One. 2010;5:e13728.
54. Maggioni M, Picotti GB, Bondiolotti GP, et al. Effects of phosphatidylserine therapy in geriatric patients with depressive disorders. Acta Psychiatr Scand. 1990;81:265-70. 